\title{
CARTESIANISMO E STORIA: DAL RIFIUTO DEL PASSATO ALLA “HISTOIRE CRITIQUE"
}

Gregorio Piaia*

SÍNTESE - No Discurso sobre o método e em outros textos Descartes vê uma oposição entre verdade e história e, por isso, recusa metodologicamente qualquer relação com o passado filosófico. Nisto é seguido por muitos de sua época, sendo que Malebranche o supera nesta reivindicação. Entretanto, a "astúcia da razão" mostra que coube exatamente à filosofia anti-histórica de Descartes o mérito de haver dado uma nova fisionomia à história filosófica que se desenvolveu no século XVI como gênero literário, e que se encontra na raiz da transformação da "história dos filósofos" em "história da filosofia".

"Conversare con gli uomini di altri secoli è quași lo stesso che viaggiare: certo, è bene saper qualcosa dei costumi dei vari popoli per giudicare meglio dei nostri, e non stimare ridicolo e irragionevole (contre raison) tutto ciò ch'è contrario alle nostre abitudini, come credono coloro che non hanno visto mai nulla; ma quando s'impiega troppo tempo a viaggiare, si diventa alla fine stranieri nel proprio paese, e così chi è troppo curioso delle cose del passato diventa, per lo più, molto ignorante di quelle presenti". ${ }^{1}$ Questa celebre similitudine del Discours de la méthode non ha soltanto un significato autobiografico, ma assurge simbolicamente a pendant critico di un altro celebre testo, la lettera che Niccolò Machiavelli, allontanato da ogni attività politica e costretto a vivere nella solitudine della campagna, scrisse a Francesco Vettori in data 10 dicembre 1513: "Venuta la sera, mi ritorno in casa, et entro nel mio scrittoio; et in sull'uscio mi spoglio quella veste cotidiana, piena di fango et di loto, e mi metto panni reali et curiali; et rivestito condecentemente entro nelle antique corti degli antiqui huomini, dove, da loro ricevuto amorevolmente, mi pasco di quel cibo, che solum è mio, et ch'io nacqui per lui; dove io non mi vergogno parlare con loro, e domandarli della ragione delle loro actioni, et quelli per loro humanità mi rispondono [...]". ${ }^{2}$

* Università di Padova.

1 DESCARTES, Discorso sul metodo, a cura di A. Carlini, XXVIII ed., Roma-Bari 1991, "Parte prima", p. 47 (R. DESCARTES, Discours de la méthode, texte et commentaire par E. Gilson, IIle éd., Paris 1962, p. 6).

2 N. MACHIAVELLI, Lettere, a cura di F. Gaeta, Milano 1961, n² 140, p. 305.

\begin{tabular}{|l|l|l|l|l|l|}
\hline VERITAS & Porto Alegre & v. 43 & $\mathrm{n}^{2} 2$ & Junho 1998 & p. 319-328 \\
\hline
\end{tabular}


Certo, la forzata solitudine del Machiavelli è diversa dalla scelta di vita solitaria e tranquilla effettuata da Cartesio. $\mathrm{Ma}$, al di là delle differenti situazioni personali, i due brani qui riportati sembrano esprimere in maniera emblematica il 'passaggio' dal clima umanistico al clima della nuova scienza e della nuova filosofia, fondate su un modello tratto dalle matematiche e non più dalle "istorie" e dalla "conversazione" con gli antichi. Eppure poche righe prima Cartesio s'era posto nella stessa lunghezza d'onda di Machiavelli, riconoscendo che "la lettura di libri buoni è come una conversazione con i loro autori, i quali furono tra le persone più illustri (les plus honnêtes gens) del passato, ed è anzi una conversazione meditata (étudiée), nella quale essi ci scoprono il meglio dei loro pensieri". ${ }^{3}$ Non è solo una benevola concessione agli antichi maestri di La Flèche, giacché il tema (già presente all'inizio della III Regula ad directionem ingenii) riaffiorerà nella prefazione alla traduzione francese dei Principia philosophiae: fra i quattro mezzi più comuni con cui si può acquistare la "saggezza" troviamo alla fine - sia pure a mo' di aggiunta - anche "la lettura non di tutti i libri, ma specie di quelli scritti da persone capaci di darci buoni insegnamenti, poiché è una specie di conversazione che noi abbiamo coi loro autori". 4 Segno che il facile cliché del puro "filosofo-matematico" non esaurisce la personalità culturale di Cartesio, che è critico verso la cultura umanistica, ma al tempo stesso ne è figlio.

L'atteggiamento anti-storico di Cartesio è comunque il dato più evidente, anche se non manca chi, come Jacques Derrida, ha individuato una paradossale storicità nell'etimologia della parola 'metodo' ("methodos, metahodos, c'est-à-dire 'suivant la route', suivant le chemin, en suivant le chemin, en chemin"), che implicherebbe una successione di momenti e quindi una struttura storica. ${ }^{5}$ In realtà la grande erudizione secentesca, che ha i.suoi simboli nella straripante biblioteca e nell'annessa Wunderkammer, in cui le reliquie antiquarie si mescolano con i reperti frutto di viaggi in paesi lontani ed esotici, sembra estranea agl'interessi di Cartesio. Étranger en son pays: è questa la paradossale ed alienante condizione in cui viene a trovarsi chi dedica troppo tempo alle lingue e ai libri degli antichi, laddove - e qui troviamo già delineata la querelle des anciens et des modernes nella sua versione filosofica - "il nostro secolo mi sembrava fiorente e ricco di buoni ingegni (en bons esprits) quanto nessuno dei precedenti". ${ }^{6}$

E vero che i libri di storia scritti dagli antichi possono aiutare a former le jugement; tuttavia essi presentano sovente elementi favolosi e anche nei testi più fedeli vengono per lo più omesse "le circostanze più basse e meno illustri; onde avviene che l'insieme appare diverso da quello che fu, e dà esca, a quanti s'ispirano a tali esempi, di cadere nelle stravaganze dei paladini dei nostri roman-

3 Discorso sul metodo, p. 46 (ed. Gilson, p. 5).

4 CARTESIO, Opere, a cura di E. Garin, Bari 1967, I, p. 13 (ed. Adam-Tannery, IX-2, p. 5). Si veda pure, nella pagina seguente, il rapido schizzo 'storico' sulle "prime cause e i veri principi", con accenni a Socrate, Platone, Aristotele, agli scettici e ai sensisti (Epicuro).

5 J. DERRIDA, La langue et le "Discours de la méthode", "Recherches sur la philosophie et le langage", 1983, 3, pp. 35-51 (36).

6 Discorso sul metodo, "parte prima", p. 45 (ed. Gilson, p. 5). 
zi, o di concepire divisamenti (desseins) superiori alle loro forze". ${ }^{7}$ Non sappiamo se Cartesio conoscesse il Don Chisciotte della Mancia, la cui seconda parte era apparsa nel 1615, ma queste assonanze gettano un ponte significativo fra il primo grande romanzo dell'età moderna e il testo che è tradizionalmente posto all'inizio della moderna filosofia: in entrambi i casi v'è la coscienza di un distacco, vuoi ironico vuoi critico, verso un passato che dalla letteratura e dalla storiografia veniva presentato come nobile ed esemplare.

Ad ogni modo le critiche di Cartesio alla storiografia del tempo, spesso animata da intenti celebrativi, sono ben azzeccate; né si può dire, quanto alla conoscenza del passato filosofico, ch'egli fosse sprovvisto d'informazioni, dal momento che dichiara di conoscere "le differenze che vi sono state in ogni tempo fra le opinioni dei più dotti". Anzi, riprendendo il detto ciceroniano (De divinatione, II, $58,119)$, egli afferma d'aver appreso, "sin dagli anni di collegio, $[\ldots]$ che non si può immaginare nulla di tanto strano e poco credibile che non sia stato detto da qualche filosofo". ${ }^{8}$ In nota a questo passo il Gilson fa presente che la medesima sentenza era stata citata da Montaigne nell'Apologie de Raymond Sebond, a proposito dei "molti volti" della filosofia, in cui si è ampiamente espressa, nel bene e nel male, l'"umana fantasia". ${ }^{9}$ Un richiamo opportuno, perché di sapore montaigniano sono anche le successive riflessioni di Cartesio - frutto del suo viaggiare - sui cosiddetti "barbari o selvaggi" (che possono in realtà ragionare meglio di noi), sull'influsso dell'ambiente in cui uno vene allevato e sul variare delle mode di abbigliamento, per cui nel nostro agire e giudicare siamo guidati non da "una conoscenza certa", bensì dall'“abitudine" (coutume) e dall"'esempio". Sicché conclude Cartesio - non potendo "scegliere nessuno le cui opinioni mi sembrassero preferibili a quelle degli altri, [...] mi trovai, si può dire, costretto a cercare di guidarmi da me stesso (je me trouvai comme contraint d'entreprendre moi-même de me conduire)". ${ }^{10}$ Una soluzione in linea con Montaigne, con la differenza - non da poco [...] - che quest'ultimo si limita alla sfera morale, mentre Cartesio (al di là della massa variegata e contrastante delle "opinioni" ch'egli ha incontrato viaggiando fra i libri e gli uomini, ossia nel tempo storico e nello spazio geografico) punta con decisione a un principio unico del sapere, su cui costruire con metodo rigoroso un edificio interamente nuovo e geometricamente armonico.

V'è dunque in Cartesio un'opposizione tra la verità, dominio delle idee chiare e distinte, e la storia, che ci presenta una congerie di opinioni slegate fra loro e prive di nessi evidenti. In quanto fondato su un metodo rigoroso, l'esercizio della filosofia si distacca nettamente dalla conoscenza storica degli antichi filosofi. "Non riusciremo mai ad essere filosofi - egli notava già nelle Regulae ad directionem ingenii, III (1627-'28) - se avremo letto tutte le argomentazioni di Platone ed Aristotele, ma senza che siamo in grado di portare giudizio sicuro intorno agli

\footnotetext{
Discorso sul metodo, pp. 46-47 (ed. Gilson, pp. 5-7).

Discorso sul metodo, "Parte seconda", p. 58 (ed. Gilson, p. 16).

Discours de la méthode, ed. Gilson, p. 178. Cfr. MONTAIGNE, Essais. Livre II, Chronologie et introduction par A. Micha, Paris 1969, cap. XII, p. 211.

Discorso sul metodo, p. 59 (ed. Gilson, p. 16).
}

10 
argomenti proposti: cosi, invero, mostreremmo di avere imparato non le scienze, ma la storia". ${ }^{11}$ È la stessa opposizione tra 'filosofia' (ovvero 'scienza') e 'storia' che si coglie negli altri protagonisti della rivoluzione scientifica del Seicento: in Bacone, con la ben nota distinzione ragione-memoria-immaginazione; in Galileo, che contrappone polemicamente ai veri filosofi gli "istorici o dottori di memoria"; 12 in Pascal, che nella prefazione al Traité sur le vide (1647) condanna l'eccessivo rispetto verso il passato e distingue due tipi di scienze: "alcune dipendono soltanto dalla memoria e sono puramente storiche: infatti si propongono di sapere quel che gli autori hanno scritto; altre dipendono solo dal ragionamento avendo per oggetto di cercare e scoprire le verità nascoste. Quelle della prima specie sono limitate, tanto quanto i libri in cui sono contenute", mentre le seconde sono destinate a progredire, poiché la ragione "è incessantemente produttiva e le sue invenzioni possono essere senza fine e senza interruzione". ${ }^{13}$

Questa sorta di Leitmotiv della nuova cultura europea è naturalmente presente nei seguaci e successori di Cartesio. L'opposizione verità-storia (che anche Hegel avrebbe enfatizzato, risolvendola però con il concetto forte di Entwicklung e con la dialettica coincidenza di filosofia e storia) è anzi più radicale in Malebranche, che contrappone a sua volta la vera filosofia o science de l'esprit alla science de mémoire di quanti si sforzano d'interpretare correttamente le dottrine di Aristotele o Platone. Difatti costoro "non sanno che storie e fatti, e non verità evidenti; e sono piuttosto degli storici che dei veri filosofi, uomini che non pensano affatto ma che sono in grado di raccontare i pensieri altrui". Il lavoro storico è giudicato pertanto superfluo di fronte all'esigenza e alla possibilità di cogliere con la ragione ciò che è vero in sé e che, in quanto tale, può prescindere tranquillamente da qualsiasi aspetto bio-bibliografico o filologico-ermeneutico: "Mi sembra che sia abbastanza inutile, per quelli che vivono presentemente, sapere se vi sia mai stato un uomo che si chiamasse Aristotele, se quest'uomo abbia scritto [effettivamente] i libri che portano il suo nome, se egli intenda una tal cosa o un'altra in un tal luogo delle sue opere; ciò non può rendere un uomo né più saggio né più felice, ma è molto importante sapere se quanto dice sia vero o falso in sé. Ė pertanto inutile sapere ciò che Aristotele ha creduto sull'immortalità dell'anima, benché sia molto utile sapere che l'anima è immortale". ${ }^{14}$

Uno spirito arguto potrebbe notare, a questo punto, che ce n'è abbastanza per proclamare il fallimento dell'attività storico-filosofica e far quindi chiuder bottega agli operatori del settore, ormai irreparabilmente 'fuori mercato'... Ma le astuzie

11 CARTESTO, Opere, a cura di E. Garin, Bari 1967, I, p. 23 (ed. Adam-Tannery, ).

12 G. GALILEI, Dialogo sopra $i$ due massimi sistemi del mondo, a cura di F. Flora, Milano 1959, p. 151. Lo stesso tema si ritrova, a sessant'anni di distanza, nel siciliano Michelangelo Fardella, che avrebbe introdotto la filosofia cartesiana nell'Università di Padova: egli definisce potius Historici quam Philosophi coloro che, propriae rationis usum deserentes, aliena mente, aliorum oculis naturam inspicere et contemplan gaudent (Universae philosophiae systema. In quo nova quadam, et extricata methodo, naturalis scientiae, et moralis fundamenta explanantur, Venetiis 1691, p. 14).

13 Grande antologia filosofica, trad. di M. F. Sciacca, XII, Milano 1968, pp. 1018-1020.

14 N. MALEBRANCHE, De la recherche de la vérité, intr. et texte établi par G. Lewis, Paris 19451946, pp. 151 e 154. 
della 'ragione storica', si sa, sono notevoli: per uno di quei paradossi di cui è intessuta la storia delle idee, è toccato proprio all'antistorica filosofia cartesiana il merito di aver dato una nuova fisionomia e un nuovo vigore a quella historia philosophica che nel corso del Seicento s'era sviluppata come un genere letterario a sé stante. In effetti, se in un primo momento il richiamo al passato appare inutile ed anzi fuorviante rispetto a un percorso che ognuno può intraprendere muovendo dall'autocoscienza, l'acquisita consapevolezza della novità e dell'eccellenza del metodo cartesiano, e quindi della rottura radicale con il passato, finisce per innescare un processo di storicizzazione: Cartesio è posto all'"inizio" di una nuova età $e$ tale periodizzamento induce al confronto con la filosofia di "ieri", che diventa oggetto di ricostruzione critica, e alla descrizione/celebrazione di un "oggi" che, grazie alla nascente idea di progresso, lascia intravvedere i contorni del "domani". V'è di più: il metodo e l'esprit stesso della filosofia cartesiana, con la sua critica alle "sètte" in nome della libertas philosophandi e con la sua aspirazione alla sistematicità, introducono una forte istanza speculativa all'interno di una visione e di una pratica storiografica le cui coordinate erano state sino ad allora fornite dall'erudizione e dalla filologia dell'età umanistico-rinascimentale, che a sua volta s'era ispirata ai modelli delle antiche biografie e dossografie. Di tale processo, che è d'importanza capitale per la nascita della 'moderna' storiografia filosofica (sorta ben prima di Hegel, con buona pace dei neo-hegeliani) richiamiamo qui alcuni degli episodi più rappresentativi. ${ }^{15}$

In primo luogo va notato che le preclusioni dei maggiori pensatori francesi del XVII secolo verso lo studio del passato non sono condivise da tutti i loro seguaci. E il caso dell'oratoriano Bernard Lamy, cartesiano e grande amico di Malebranche, che riconosce la validità - per lo meno sul piano didattico - di un approccio storico alla filosofia. Nella sezione degli Entretiens sur les sciences (1684). dedicata ai filosofi il Lamy riprende la distinzione malebranchiana tra la filosofia, fondata sulla ragione di cui ogni uomo dispone, e la storia, fondata sull'autorità e sui libri, che in gran parte "sono un ostacolo alla vera scienza". Subito dopo, però, egli osserva realisticamente che i libri non sono inutili, ma possono servire da guida per coloro che iniziano lo studio della filosofia, "giacché per due o tre persone che, essendosi disfatte di tutte le opinioni che avevano appreso altrove e rinunciando ai libri, hanno attinto con successo al fondo di loro stesse la verità, ve ne sono un numero infinito che, per aver voluto camminare senza guida, si sono fuorviate e sono cadute in mille fantasticherie". Coloro che studiano nelle università e non sono in grado di usare correttamente la loro ragione dovrebbero pertanto fare uso della memoria per conservare i principi elaborati dai "filosofi illustri". ${ }^{16}$

La corporazione degli storici della filosofia potrebbe tirare un mezzo respiro di sollievo, poiché è riconosciuta la consolante (ma non esaltante) possibilità d'essere richiamati in servizio nei ranghi inferiori e con compiti esclusivamente didattici e

15 Per una trattazione più ampia e dettagliata si rinvia al vol. II dell'opera in più tomi Storia delle storie generali della_filosofia, la cui pubblicazione (a cura di Giovanni Santinello e di chi scrive) è ancora in corso: F. BOTTTN - M. LONGO - G. PIAIA, Dall'età cartesiana a Brucker, Brescia, Ed. La Scuola, 1979, da cui abbiamo ricavato alcuni estratti.

16

B. LAMY, Entretiens sur les sciences, éd. critique par F. Girbal et P. Clair, Paris 1966, pp. 237-239. 
della 'ragione storica', si sa, sono notevoli: per uno di quei paradossi di cui è intessuta la storia delle idee, è toccato proprio all'antistorica filosofia cartesiana il merito di aver dato una nuova fisionomia e un nuovo vigore a quella historia philosophica che nel corso del Seicento s'era sviluppata come un genere letterario a sé stante. In effetti, se in un primo momento il richiamo al passato appare inutile ed anzi fuorviante rispetto a un percorso che ognuno può intraprendere muovendo dall'autocoscienza, l'acquisita consapevolezza della novità e dell'eccellenza del metodo cartesiano, e quindi della rottura radicale con il passato, finisce per innescare un processo di storicizzazione: Cartesio è posto all"'inizio" di una nuova età e tale periodizzamento induce al confronto con la filosofia di "ieri", che diventa oggetto di ricostruzione critica, e alla descrizione/celebrazione di un "oggi" che, grazie alla nascente idea di progresso, lascia intravvedere i contorni del "domani". V'è di più: il metodo e l'esprit stesso della filosofia cartesiana, con la sua critica alle "sètte" in nome della libertas philosophandi e con la sua aspirazione alla sistematicità, introducono una forte istanza speculativa all'interno di una visione e di una pratica storiografica le cui coordinate erano state sino ad allora fornite dall'erudizione e dalla filologia dell'età umanistico-rinascimentale, che a sua volta s'era ispirata ai modelli delle antiche biografie e dossografie. Di tale processo, che è d'importanza capitale per la nascita della 'moderna' storiografia filosofica (sorta ben prima di Hegel, con buona pace dei neo-hegeliani) richiamiamo qui alcuni degli episodi più rappresentativi. ${ }^{15}$

In primo luogo va notato che le preclusioni dei maggiori pensatori francesi del XVII secolo verso lo studio del passato non sono condivise da tutti i loro seguaci. E il caso dell'oratoriano Bernard Lamy, cartesiano e grande amico di Malebranche, che riconosce la validità - per lo meno sul piano didattico - di un approccio storico alla filosofia. Nella sezione degli Entretiens sur les sciences (1684).dedicata ai filosofi il Lamy riprende la distinzione malebranchiana tra la filosofia, fondata sulla ragione di cui ogni uomo dispone, e la storia, fondata sull'autorità e sui libri, che in gran parte "sono un ostacolo alla vera scienza". Subito dopo, però, egli osserva realisticamente che i libri non sono inutili, ma possono servire da guida per coloro che iniziano lo studio della filosofia, "giacché per due o tre persone che, essendosi disfatte di tutte le opinioni che avevano appreso altrove e rinunciando ai libri, hanno attinto con successo al fondo di loro stesse la verità, ve ne sono un numero infinito che, per aver voluto camminare senza guida, si sono fuorviate e sono cadute in mille fantasticherie". Coloro che studiano nelle università e non sono in grado di usare correttamente la loro ragione dovrebbero pertanto fare uso della memoria per conservare i principi elaborati dai "filosofi illustri". ${ }^{16}$

La corporazione degli storici della filosofia potrebbe tirare un mezzo respiro di sollievo, poiché è riconosciuta la consolante (ma non esaltante) possibilità d'essere richiamati in servizio nei ranghi inferiori e con compiti esclusivamente didattici e

15 Per una trattazione più ampia e dettagliata si rinvia al vol. II dell'opera in più tomi Storia delle storie generali della_filosofia, la cui pubblicazione (a cura di Giovanni Santinello e di chi scrive) è ancora in corso: F. BOTTTN - M. LONGO - G. PIAIA, Dall'età cartesiana a Brucker, Brescia, Ed. La Scuola, 1979, da cui abbiamo ricavato alcuni estratti.

16 B. LAMY, Entretiens sur les sciences, éd. critique par F. Girbal et P. Clair, Paris 1966, pp. 237-239. 
propedeutici (l'insegnamento dell'historia philosophica, a mo' di surrogato, a coloro che non sono in grado di praticare autonomamente l'esercizio della filosofia...). Una svolta effettiva nell'atteggiamento del cartesianismo verso il passato si verifica però di li a pochi anni, quando gli editori olandesi giudicarono opportuno arricchire con un breve "discorso sulla filosofia antica e moderna" la terza edizione della summa cartesiana di Pierre-Sylvain Régis: Cours entier de philosophie, ou système général selon les principes de $M$. Descartes, contenant la logique, la métaphysique, la physique, et la morale. Dernière édition, enrichie d'un très grand nombre de figures, et augmentée d'un Discours sur l'histoire de la philosophie ancienne et moderne, où l'on fait en abrégé l'histoire de cette science, Amsterdam 1691.

La prima edizione dell'opera (con titolo alquanto diverso) risale appena all'anno addietro, segno evidente del successo ottenuto presso un pubblico che s'era aperto alle novità cartesiane. Ma nel corso del Seicento la cultura olandese aveva anche sviluppato uno specifico interesse per le indagini storico-filosofiche, in particolare con gli Historiae philosophicae libri VII (1655) di Georg Horn, il De philosophorum sectis (1657) di Johannes Gerhard Vossius e l'Historia philosophica (1674) di Abraham de Grau. ${ }^{17}$ Non stupisce allora che, di fronte al successo registrato dall'opera del Régis, si sia pensato d'inserirvi un'introduzione storica, analogamente con quanto avveniva in taluni manuali di filosofia in lingua latina destinati alle scuole, come il Cursus Conimbricensis. Questo non significa però un cedimento alla tradizione, ché il Discours sur la philosophie ancienne et moderne (apparso anonimo ma steso dall'emigrato calvinista Pierre Coste, futuro amico e traduttore di John Locke) si caratterizza per una chiara e provocatoria impostazione cartesiana, che sovverte i canoni della storiografia di matrice umanistica ed assume una posizione assai netta nella "querelle des anciens et des modernes", ovvero nella disputa tra i peripatetici e i propugnatori della "nouvelle philosophie".

In larvata polemica con le Réflexions sur la philosophie ancienne et moderne, et sur l'usage qu'on en doit faire pour la religion (1676) del gesuita René Rapin, ammiratore degli antichi e seguace di Aristotele, l'autore del Discours contesta infatti la presunta superiorità degli anciens. Ciò vale soprattutto per la filosofia: "poiché è una scienza che dipende dalla precisione del ragionamento - il quale non si perfeziona d'un colpo - e che è composta da un numero infinito d'intuizioni e aiutata da esperienze che il caso produce comunemente e non mena a proposito, è evidente che i primi filosofi debbono aver lasciato parecchie cose da fare a quelli che vengono dopo di loro". ${ }^{18}$ Questo senso dello sviluppo progressivo delle umane conoscenze induce Pierre Coste ad affermare che la filosofia si trova oggi "al più alto grado di perfezione", e ciò grazie all'impiego di un metodo diverso, fondato sulla ragione e non sull'autorità. I filosofi moderni si distinguono per la "nouvelle manière de philosopher", tant'è vero che Cartesio, "grazie a un metodo ch'era

17 Cfr. il contributo di L. MALUSA, Le prime storie della filosofia in Inghilterra e nei Paesi Bassi, in Storia delle storie generali della filosofia, I: Dalle origini rinascimentali alla "historia philosophica", Brescia 1981, pp. 217-300.

18 [P. COSTE], Discours sur la philosophie ancienne et moderne, in P.S. RĖGIS, Cours entier de philosophie, Amsterdam 1691 (rist. New York 1971), p. [2]). 
stato conosciuto solo imperfettamente prima di lui, ha scoperto più verità di quante ne siano state scoperte in tutti i secoli precedenti". ${ }^{19}$

È per l'appunto il metodo o "manière de raisonner" che costituisce il primo dei tre criteri di giudizio su cui il Coste fonda la comparaison tra la filosofia antica e la moderna (gli altri due si riferiscono al grado di estensione delle conoscenze "particolari", relative cioè alla fisica, e alla disponibilità di strumenti scientifici): "Per quanto concerne, in primo luogo, la maniera di ragionare, i filosofi moderni superano visibilmente gli antichi, giacchè, mentre questi ultimi non ragionavano il più sovente che su idee vaghe e su principi molto confusi, quelli si impongono di ragionare su idee chiare e distinte, e di passare dalle cose semplici e facili a comprendersi a quelle composte e meno conosciute. Quand'anche la filosofia di Cartesio non fosse servita che ad introdurre questo nuovo metodo di ragionare lè implicito il riferimento alle forti critiche di cui era stata oggetto la fisica cartesiana, rivelatasi ben presto la parte più caduca dell'intero sistema di pensierol, essa sarebbe più degna di stima di tutta la filosofia degli antichi". ${ }^{20}$ Quest'ultima considerazione va evidenziata, perché denota un tentativo di storicizzare la posizione e il ruolo di Cartesio. Il prezzo pagato è certamente alto (la 'resa incondizionata' di tutta la filosofia antica, Aristotele compreso, ai moderni), ma è per tale via che l'historia philosophica, che sembrava destinata al confino negli aridi territori dell'erudizione, acquista diritto di cittadinanza nel regno giovane e in piena espansione della nouvelle philosophie.

Un altro apporto in tal senso, sul piano teorico ben più sostanzioso del giovanile (e giornalistico) Discours di Pierre Coste, viene da una delle maggiori realizzazioni del metodo e dello spirito cartesiano: quella Logique de Port-Royal ou Art de penser di Antoine Amauld e Pierre Nicole che godette di enorme fortuna in tutta Europa ed influi profondamente sugli sviluppi successivi della logica e della gnoseologia. Può apparire strano che una simile opera abbia influito anche sul modo d'intendere la storia della filosofa, che con la sua estrema varietà di opinioni è agli antipodi di un lineare discorso logico, ma il movimento delle idee - come già s'è visto - imbocca strade a volte impensabili o comunque imprevedibili. In questo caso è il riconoscimento di una verità della storia accanto alle verità di ragione e a quelle di fede, espresso con chiarezza nell'ultima parte dell'Art de penser, ${ }^{21}$ ad offrire il presupposto per una vera e propria fondazione epistemologica del sapere storico, e quindi anche storico-filosofico. È quanto sarà attuato da Pierre Bayle, che a buon diritto si può considerare un aderente al cartesianismo, sia pure con

19 Ivi, pp. [36]-[37].

20 Ivi, p. [44].

21 La logique ou l'Art de penser, Paris 1662 (rist. Genève 1972), pp. 433-434: “Et neanmoins [...] il y a des choses que nous ne connoissons que par une foy humaine, que nous devons tenir pour aussi certaines et aussi indubitables, que si nous en avions les demonstrations mathematiques.[...] I faudroit de mesme avoir perdu le sens, pour douter si jamais Cesar, Pompée, Ciceron, Virgile ont esté; et si ce ne sont point des personnages feints, come ceux des Amadis [...]"; e qui il richiamo ad Amadigi di Gaula, protagonista di un celebre romanzo cavalleresco spagnolo, ci riporta al cartesiano accenno alle "extravagances des paladins de nos romans"... 
tendenze eclettiche e con le ben note propensioni scettiche (un "cartesiano minor", com'ebbe a definirlo Elisabeth Labrousse ). ${ }^{22}$

Nelle ultime pagine del Projet et fragmens d'un Dictionnaire critique (Rotterdam 1692), diretto a preparare il terreno alla prima edizione del monumentale Dictionnaire historique et critique (1697), il Bayle rivendica la validità e l'autonomia della conoscenza storica, attribuendo alle verità di fatto un proprio grado di certezza, superiore a quello delle stesse verità di ordine matematico. È opportuno riportare per esteso questo abbozzo di una teoria della storiografia, che segna il superamento della preclusione cartesiana verso il mondo storico e l'estensione ad esso del principio dell'evidenza, staccato dal suo iniziale contesto puramente geometrico e metafisico. "Se $\mathrm{mi}$ si dice che i teoremi più astratti dell'algebra sono molto utili alla vita, perché rendono la mente dell'uomo più adatta a perfezionare certe arti, dirò che la ricerca scrupolosa di tutti i fatti storici è capace di produrre beni grandissimi. [...] Mi si obietterà forse che nelle matematiche anche ciò che appare più astratto e infruttuoso ha almeno il vantaggio di condurci a verità indubitabili, mentre le discussioni storiche e le ricerche intomo ai fatti umani, oltre a lasciarci nelle tenebre, sono semi di nuove discordie. Ma chi afferma queste cose, dà prova di scarsa prudenza. Sostengo che le verità storiche possono essere portate a un grado di certezza ancor più indubitabile di quello cui pervengono le verità geometriche; beninteso, a patto che si considerino questi due tipi di verità secondo il grado di certezza che è loro proprio. Mi spiego. Nelle dispute che sorgono fra gli storici per sapere se un certo principe ha regnato prima o dopo di un altro, si suppone dalle due parti che un fatto abbia tutta la realtà e l'esistenza di cui è capace al di fuori del nostro intelletto, purché non sia della natura di quelli riferiti dall'Ariosto o da altri narratori di fantasia, e non si tiene alcun conto delle difficoltà delle quali si servono i pirroniani per farci dubitare se le cose che ci paiono esistere esistano realmente al di fuori del nostro pensiero. Cosi un fatto storico raggiunge il più alto grado di certezza che ad esso conviene, non appena si sia accertata la sua esistenza apparente [...]" ${ }^{23}$

Questa concezione generale della conoscenza. storica è significativamente applicata anche all'ambito storico-filosofico (nel Dictionnaire bayliano 104 sono gli articoli - spesso assai lunghi e densi di riflessioni - dedicati ai filosofi antichi e moderni). Ė il caso dell'articolo su Epicuro, ove il Bayle, dopo aver riportato un passo del De finibus in cui Cicerone sottolinea positivamente il legame di amicizia fra gli epicurei, coglie lo spunto per riproporre la celebre tesi dell'"ateo virtuoso". A chi era solito presentare gli epicurei come un poco di buono, deducendo tale giudizio dalle loro tesi sulla provvidenza e sul piacere, egli obietta nichiamandosi al primato della verità di fatto: "Tutte queste belle dottrine non sono forse confutate da quest'unico passo di Cicerone? Una verità di fatto, come quella che Cicerone ci ha attestato, non capovolge forse cento volumi di ragionamenti speculativi?" ${ }^{24}$

22 Cfr. E. LABROUSSE, Pierre Bayle, La Haye 1963-1964, II, pp. 41-44.

23 P. BAYLE, Dictionnaire historique et critique, Amsterdam... 1740, IV, p. 613 (tr. it. di M. Ghio in Grande antologia filosofica, XIV, Milano 1968, p. 444).

24 BAYLE, Dictionnaire, II, p. 365a (art. Epicure, rem. D). 
Rifondato sulla base del pieno valore epistemico delle "verità di fatto", lo studio del passato filosofico diviene cosi - per la natura stessa del suo oggetto - il campo privilegiato di analisi e di giudizio, in cui trova piena applicazione il potenziale critico del metodo cartesiano. Certo il Bayle - come già notava Cassirer - non ha una visione progressiva e teleologica della storia della filosofia, che in lui si risolve invece in una "conoscenza dei particolari [che] non porta alla vera comprensione del tutto, ma distrugge invece ogni speranza di una tale comprensione". ${ }^{25} \mathrm{Ma}$ l'esasperata e talora disperante indagine del Bayle su un particolare biobibliografico o su un tema dottrinale, in nome della coerenza interna sia ai fatti storici sia alle idee filosofiche e teologiche, è anch'essa riconducibile al rigore e alla lucidità di Cartesio, resi ancor più affinati dai contatti con il pirronismo storico; ed è dalla lezione di metodo impartita dal "Dizionario storico e critico" che si sviluppa quella histoire critique (detta pure raisonnée o philosophique) che introduce un profondo rinnovamento nella tradizione storiografica, anche e soprattutto nel campo filosofico: non più semplice registrazione di dati, bensi analisi e valutazione (jugement, iudicium), e quindi aperta denuncia degli errori, per cui il termine critique, che tradizionalmente era riferito all'ars critica, ossia alla filologia, viene ad assumere il significato, ancor oggi corrente, di critica razionale e filosofica.

Histoire critique de la philosophie: così André-François Boureau-Deslandes, che in gioventù ebbe in simpatia le dottrine di Malebranche, intitolerà la sua opera (1737), in cui il Leitmotiv della contrapposizione storici-filosofi viene applicato all'interno della stessa storiografia. "Fra gli scrittori della storia filosofica - egli nileva in un Eclaircissement all'inizio dell'opera - gli uni hanno lavorato senza scelta, senza discernimento, più da compilatori che raccolgono che da censori che giudicano. Essi hanno riferito i pensieri degli altri, e non si sono curati abbastanza di pensare essi stessi. [...] In effetti a che giova mettere in mostra agli occhi del pubblico le dottrine di Pitagora, di Platone, d'Aristotele e d'Epicuro, se al tempo stesso non si mette in chiaro ciò che vi è di lodevole o di riprovevole in tali dottrine? Se non si penetrano i motivi che hanno dato loro la nascita, e le illusioni che possono far sorgere nella mente, e le sorprese che fanno al cuore?"26 (e qui va evidenziata l'attenzione che il Boureau-Deslandes, al pari di tanti altri autori settecenteschi, riserva alla "sensibilità" e aile raisons du coeur, che vengono pascalianamente ad integrare le ragioni della mente).

Su questa linea, ma con un impianto di gran lunga più sistematico, si muove la massiccia Historia critica philosophiae di Johann Jakob Brucker (1742-1744), la maggior produzione storico-filosofica del primo Settecento, che Victor Cousin considerò come l'ultima e più matura espressione del cartesianismo. ${ }^{27}$ È un giudizio che pecca di schematicità, dal momento che il Brucker, allievo del Buddeus, si professava eclettico e per lui è semmai Leibniz, e non Cartesio, la figura centrale

25 E. CASSIRER, La filosofia dell'Mluminismo, tr. it., Firenze 1964, p. 287.

26 A.-F. BOUREAU-DESLANDES, Histoire critique de la philosophie [...], Ile éd., Amsterdam 1756, I, pp. XVII-XVIII.

27 V. COUSIN, Cours de philosophie. Introduction à l'histoire de la philosophie [1828], texte revu par P. Vermeren, Paris 1991, pp. 312-316. 
del pensiero moderno. ${ }^{28} \mathrm{E}$ un fatto, tuttavia, che senza l'apporto del razionalismo cartesiano l'evoluzione verso la storia 'critica' della filosofia non avrebbe avuto luogo. In sintesi, Cartesio sembrava aveva fondato la possibilità di un filosofare rigoroso a prezzo della storia; in realtà, in una prospettiva di media durata, il suo rifiuto del passato non comporta la fine della storiografia come pratica culturale o la sua messa ai margini, bensi - per riprendere un tema caro a Leibniz $-{ }^{29}$ la trasformazione della tradizionale "storia dei filosofi" nella moderna "storia della filosofia". È anche questo un esito - e non fra i meno rilevanti - della 'rivoluzione' cartesiana.

28 Cfr. J.J. BRUCKER, Historia critica philosophiae [...], Lipsiae 1742-1744, V, 251.

29 G.W. LEIBNIZ, Die philosophischen Schniften, hrsg. v. C.J. Gerhardt, Berlin 1875-1890 (rist. Hildesheim - New York 1978), IV, p. 162. La svolta teorica e metodologica segnata dal passaggio da una "storia dei filosofi" a una "storia della filosofia" sarebbe stata sottolineata - oltre due secoli dopo Leibniz - da Eduard Zeller nel manifesto (Die Geschichte der Philosophie, ihre Ziele und Wege) che inaugurò l'"Archiv für Geschichte der Philosophie", 1 (1888), p. 7. 